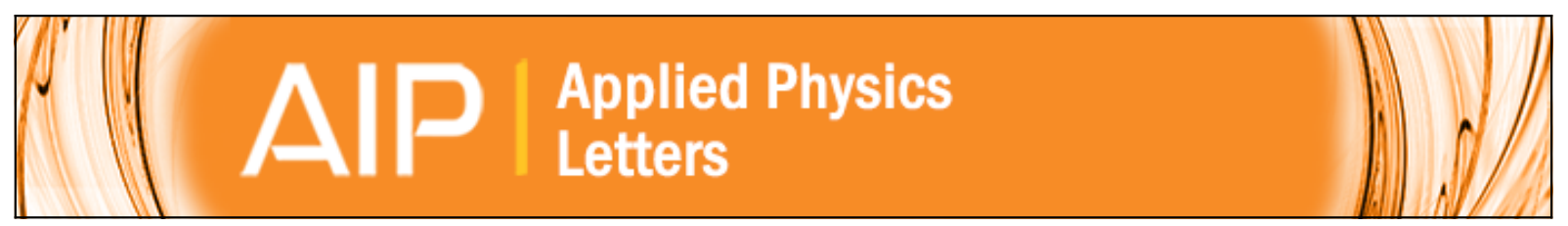

\title{
Enhanced thermoelectric figure of merit in strained Tl-doped Bi2Se3
}

Y. Saeed, N. Singh, and U. Schwingenschlögl

Citation: Applied Physics Letters 105, 031915 (2014); doi: 10.1063/1.4890858

View online: http://dx.doi.org/10.1063/1.4890858

View Table of Contents: http://scitation.aip.org/content/aip/journal/apl/105/3?ver=pdfcov

Published by the AIP Publishing

\section{Articles you may be interested in}

Enhanced thermoelectric performance of nanostructured topological insulator $\mathrm{Bi} 2 \mathrm{Se} 3$

Appl. Phys. Lett. 106, 053102 (2015); 10.1063/1.4907252

Molecular beam epitaxial growth of Bi2Se3 nanowires and nanoflakes

Appl. Phys. Lett. 105, 133109 (2014); 10.1063/1.4896966

Thermoelectric properties of topological insulator $\mathrm{Bi} 2 \mathrm{Te} 3, \mathrm{Sb} 2 \mathrm{Te} 3$, and $\mathrm{Bi} 2 \mathrm{Se} 3$ thin film quantum wells Appl. Phys. Lett. 105, 123117 (2014); 10.1063/1.4896680

Thickness and strain effects on the thermoelectric transport in nanostructured Bi2Se3

Appl. Phys. Lett. 104, 033105 (2014); 10.1063/1.4862923

Phonon spectrum and bonding properties of Bi2Se3: Role of strong spin-orbit interaction Appl. Phys. Lett. 100, 082109 (2012); 10.1063/1.3689759

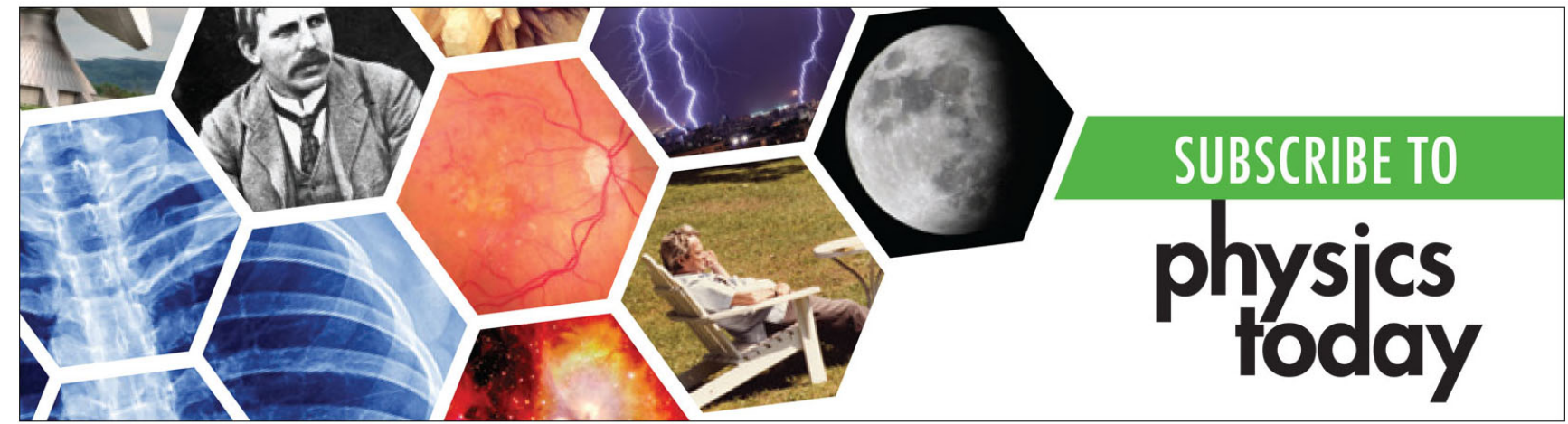




\title{
Enhanced thermoelectric figure of merit in strained TI-doped $\mathrm{Bi}_{2} \mathrm{Se}_{3}$
}

\author{
Y. Saeed, N. Singh, and U. Schwingenschlögl $\left.\right|^{\text {a) }}$ \\ PSE Division, KAUST, Thuwal 23955-6900, Kingdom of Saudi Arabia
}

(Received 14 March 2014; accepted 10 July 2014; published online 23 July 2014)

\begin{abstract}
We explain recent experimental findings on Tl-doped $\mathrm{Bi}_{2} \mathrm{Se}_{3}$ by determining the electronic and transport properties by first-principles calculations and semi-classical Boltzmann theory. Though Tl-doping introduces a momentum-dependent spin-orbit splitting, the effective mass of the carriers is essentially not modified, while the band gap is reduced. $\mathrm{Tl}$ is found to be exceptional in this respect as other dopants modify the dispersion, which compromises thermoelectricity. Moreover, we demonstrate that only after Tl-doping strain becomes an efficient tool for enhancing the thermoelectric performance. A high figure of merit of 0.86 is obtained for strong $p$-doping $\left(7 \times 10^{20} \mathrm{~cm}^{-3}\right.$, maximal power factor) at $500 \mathrm{~K}$ under $2 \%$ tensile strain. (C) 2014 AIP Publishing LLC. [http://dx.doi.org/10.1063/1.4890858]
\end{abstract}

Thermoelectric materials have been extensively studied for key applications such as the conversion of waste heat into electricity, with great potential to contribute to a solution of the present energy and environment problems. The efficiency of thermoelectric devices is determined by the dimensionless figure of merit $\sigma S^{2} T / \kappa$, where $\sigma$ is the electrical conductivity, $S$ is the Seebeck coefficient, $T$ is the temperature, and $\kappa$ is the thermal conductivity. The latter comprises lattice $\left(\kappa_{l}\right)$ and electronic $\left(\kappa_{e}\right)$ contributions, $\kappa=\kappa_{l}+\kappa_{e}$. In order to achieve a high efficiency, the thermoelectric material, in general, should be a good electrical and poor thermal conductor and, at the same time, possess a high $S$. For a literature review, see, for example, Ref. 1 and the references therein. Alloying, defects, functionalization, and the application of strain are effective and frequently used approaches to improve thermoelectric properties, because the phonon scattering can be enhanced and the thermal conductivity consequently reduced. ${ }^{2-5}$

$\mathrm{Bi}_{2} \mathrm{Se}_{3}$ has a layered tetradymite-type rhombohedral structure $(R \overline{3} m$, no. 167$)$, often described in a hexagonal representation with 15 atoms per unit cell and lattice parameters $a=4.143 \AA$ and $c=28.636 \AA^{6}{ }^{6}$ Together with its isostructural sister compounds $\mathrm{Bi}_{2} \mathrm{Te}_{3}$ and $\mathrm{Sb}_{2} \mathrm{Te}_{3}$, it has potential in the fields of topological insulators and thermoelectrics, with an energy gap of $0.3 \mathrm{eV}$ in the bulk. For enhancing the electrical conductivity and reducing the thermal conductivity of bulk $\mathrm{Bi}_{2} \mathrm{Se}_{3}$, the introduction of point defects and doping by $\mathrm{Ca}, \mathrm{Sb}$, and $\mathrm{Cu}$ have been put forward..$^{7-10}$ On the other hand, only few studies have dealt with the effect of doping by group IIIA elements, for example, $\mathrm{Ga}^{11}$ and $\mathrm{In}^{12}$ on the thermal transport. The importance of the van der Waals interaction for the thermoelectric properties of bulk $\mathrm{Bi}_{2} \mathrm{Se}_{3}$ and $\mathrm{Bi}_{2} \mathrm{Te}_{3}$ with and without strain has been emphasized in Refs. 13 and 14.

Tl-doping in topological insulators has been found to be a promising approach to enhance the thermoelectricity. The impact in the case of $\mathrm{Bi}_{2} \mathrm{Se}_{3}$ has been addressed in Ref. 15 and a linear increase of the power factor with the $\mathrm{Tl}$ concentration has been reported. Recently, the thermoelectric properties of Tl-doped $\mathrm{Bi}_{2} \mathrm{Te}_{3}$ with $\mathrm{Tl}$ concentrations of $0 \leq x \leq 0.3$ have been studied and positive effects on the

a)udo.schwingenschlog1@kaust.edu.sa. Tel.: +966(0)544700080. performance observed. ${ }^{16}$ In order to enable a systematic optimization, it is important to gain physical insight into the modifications of the electronic band structure induced by Tl-doping, which is achieved in the present work. In addition, we will study strain as a means of enhancing the figure of merit, obtaining very promising results.

We model the effect of Tl-doping on $\mathrm{Bi}_{2} \mathrm{Se}_{3}$ by addressing the system $\mathrm{Bi}_{1.67} \mathrm{Tl}_{0.33} \mathrm{Se}_{3}$ by first-principles calculations. We fully optimize the structural parameters and relax the atomic positions, taking into account the van der Waals interaction by means of the B97-D functional. ${ }^{17,18}$ The plane wave energy cutoff is set to $544 \mathrm{eV}$ and a Monkhorst-Pack $8 \times 8 \times 8 \mathrm{k}$-mesh is used for the Brillouin zone integrations. Moreover, the Perdew-Burke-Ernzerhof parametrization is employed and spin orbit coupling is included. An energy convergence of $10^{-5} \mathrm{eV}$ and a force convergence of $0.025 \mathrm{eV} / \AA$ p provide sufficient accuracy.

The transport behavior is studied using the BoltzTraP code, ${ }^{19}$ which requires a dense $\mathrm{k}$-mesh of $50 \times 50 \times 20$ points. This approach previously has led to accurate results for various types of thermoelectric materials. ${ }^{20-22}$ From the transport function $\sigma(E)=\operatorname{DOS}(E) v^{2}(E) \tau(E)$, the electrical conductivity

$$
\sigma(T)=-\int_{-\infty}^{\infty} \sigma(E) \frac{d f(E-\mu)}{d E} d E
$$

and Seebeck coefficient

$$
S(T)=-\frac{k_{B}}{e \sigma(T)} \int_{-\infty}^{\infty} \sigma(E) \frac{E-\mu}{T} \frac{d f(E-\mu)}{d E} d E
$$

are obtained, where $f$ is the Fermi function, $\tau(E)$ is the scattering time, $v(E)$ is the Fermi velocity, $\mu$ is the chemical potential, and $T$ is the temperature. Within the constant relaxation time approximation, $S$ can be obtained directly from the electronic band structure. Previous theoretical studies of $\mathrm{Bi}_{2} \mathrm{Te}_{3}$ and related compounds employing this approximation found good agreement of the predictions with experiment. ${ }^{23}$ Since it is known that strain engineering of $\mathrm{Sb}_{2} \mathrm{Te}_{3}$ can increase the power factor by tuning $\sigma$ and $S,{ }^{24,25}$ we address in the following the thermoelectric properties of $\mathrm{Bi}_{1.67} \mathrm{Tl}_{0.33} \mathrm{Se}_{3}$ under in-plane strain. 


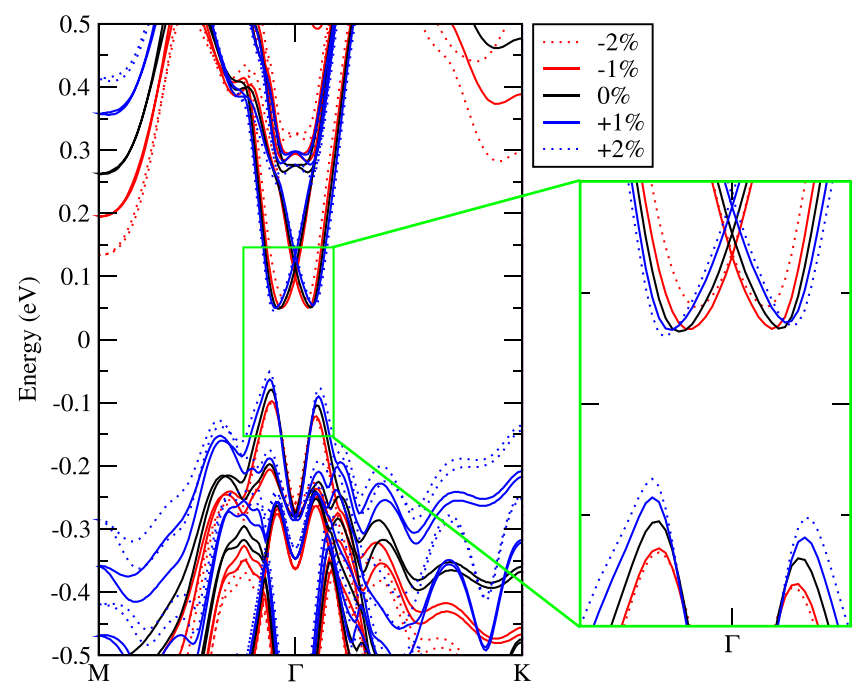

FIG. 1. Band structure obtained for (black) zero, compressive (negative sign, red), and tensile (positive sign, blue) strain.

For bulk $\mathrm{Bi}_{2} \mathrm{Se}_{3}$, our optimized lattice parameters ( $a=b=4.15 \AA$ and $c=28.28 \AA$ ), the distance between adjacent quintuple layers $(2.40 \AA)$, the thickness of the quintuple layer (7.03 $\AA$ ), and the band gap $(312 \mathrm{meV})$ are in agreement with experiment. ${ }^{6,26} \mathrm{We}$ replace one $\mathrm{Bi}$ atom with $\mathrm{Tl}$ to obtain $\mathrm{Bi}_{1.67} \mathrm{Tl}_{0.33} \mathrm{Se}_{3}$ and then re-optimize the lattice parameters, which results in a considerable volume reduction $(a=b=4.14 \AA$ and $c=27.90 \AA)$. Furthermore, we apply in-plane biaxial strain by modifying the $a$ and $b$ lattice constants, optimizing the $c$ lattice constant and relaxing the atomic positions. For $2 \%$ compressive strain $c$ grows to $29.05 \AA$, whereas it decreases to $26.81 \AA$ for $2 \%$ tensile strain.
The band structure of unstrained $\mathrm{Bi}_{1.67} \mathrm{Tl}_{0.33} \mathrm{Se}_{3}$ is given in Fig. 1 by black lines. The indirect band gap amounts to $158 \mathrm{meV}$, which is about half of the direct band gap of bulk $\mathrm{Bi}_{2} \mathrm{Se}_{3}(300 \mathrm{meV})$. Comparison to the band structure of $\mathrm{Bi}_{2} \mathrm{Se}_{3}$ reveals that Tl-doping does not modify the effective masses of the charge carriers at band edges, whereas the band gap is substantially reduced. In addition, the doping breaks the inversion symmetry, which results in a momentum-dependent spin-orbit splitting (Rasbha spin splitting) at the $\Gamma$ point (as a consequence of the potential gradient in the direction perpendicular to the atomic layers). The band structure of strained $\mathrm{Bi}_{1.67} \mathrm{Tl}_{0.33} \mathrm{Se}_{3}$ is also shown in Fig. 1, demonstrating significant strain effects particularly in the vicinity of the Fermi energy. Besides shifting the conduction band minimum and valence band maximum in energy, strain also shifts them away from the $\Gamma$ point (see the inset in Fig. 1), i.e., the Rasbha spin splitting and consequently the thermoelectric properties are altered. The band gap increases under compressive strain $(195 \mathrm{meV}$ for $1 \%$ and $202 \mathrm{meV}$ for $2 \%$ strain) and decreases under tensile strain (127 meV for $1 \%$ and $104 \mathrm{meV}$ for $2 \%$ strain), both monotonously. Under compressive strain the conduction band at the $M$ point shifts to lower energy, whereas under tensile strain the valence band at the $\mathrm{K}$ point shifts to higher energy. By these variations $\sigma$ and $S$ are tuned, which cannot be achieved by modifying the $\mathrm{Tl}$ concentration.

Optimization of the carrier concentration is often fruitful to enhance the figure of merit of a thermoelectric material. $^{27,28}$ The room temperature $(300 \mathrm{~K}) S, \sigma / \tau$, and $S^{2} \sigma / \tau$ of $\mathrm{Bi}_{1.67} \mathrm{Tl}_{0.33} \mathrm{Se}_{3}$ as a function of the carrier concentration are shown in Fig. 2 for both $n$ - and $p$-doping and for different values of the strain. An experimental room temperature $S=-66 \mu \mathrm{V} / \mathrm{K}$ has been reported for $x=0.1$ together with a
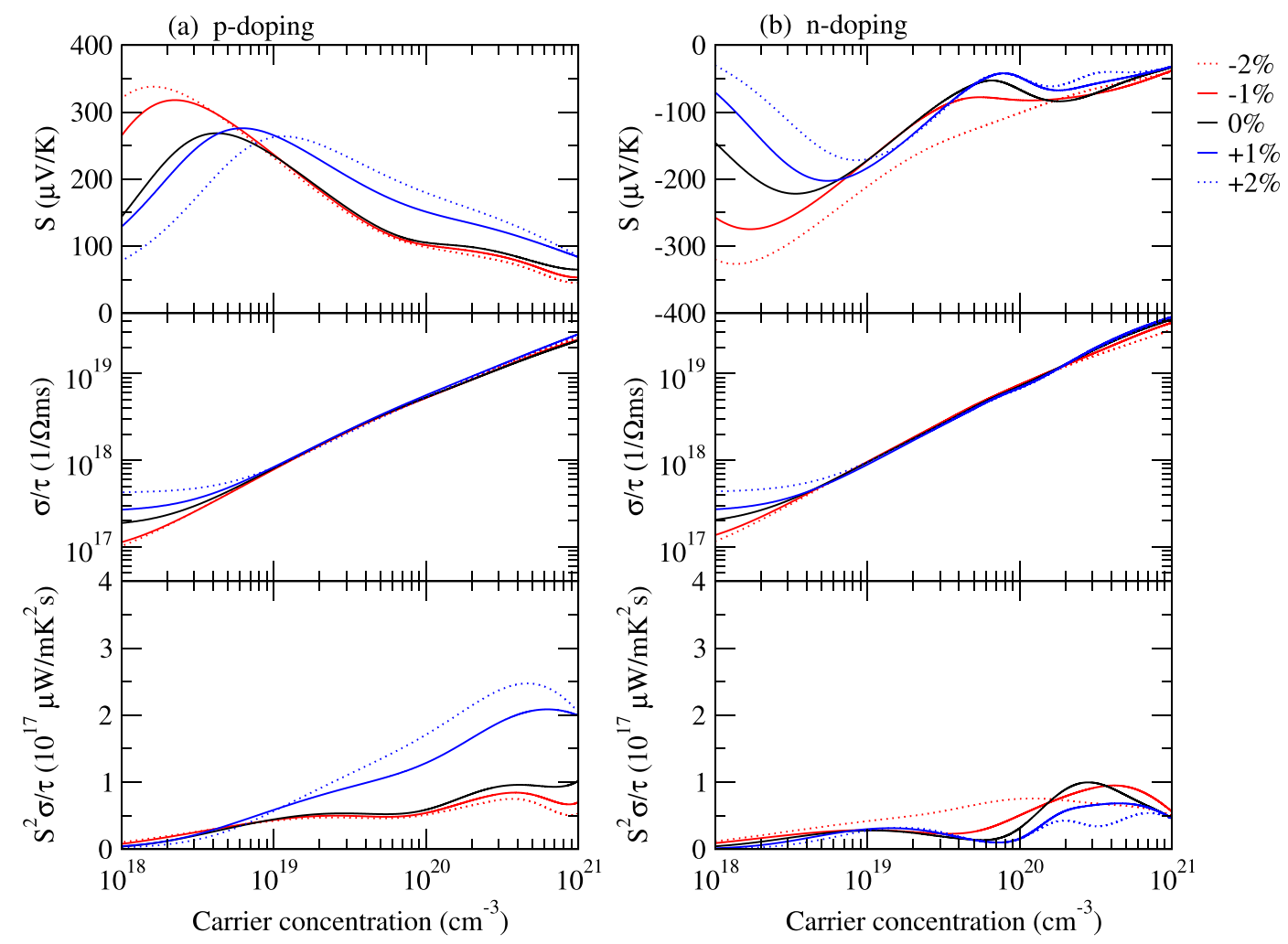

FIG. 2. Room temperature: $S, \sigma / \tau$, and $S^{2} \sigma / \tau$ under strain as a function of the carrier concentration. 
carrier concentration of $2 \times 10^{19} \mathrm{~cm}^{-3} \cdot{ }^{15}$ Our value at the same temperature and carrier concentration is $-114 \mu \mathrm{V} / \mathrm{K}$ for the unstrained system, which is higher than the experimental value because of the higher Tl-doping of $x=0.33$. For $2 \%$ compressive strain, the maximal values of $S$ are found at hole/electron concentrations of about $1.5 \times 10^{18} \mathrm{~cm}^{-3}$. In general, for low carrier concentrations the magnitude of $S$ follows the variation of the band gap, whereas the maxima in Fig. 2 shift towards higher carrier concentrations (as expected, since the band gap decreases). Above carrier concentrations of $10^{19} \mathrm{~cm}^{-3}$ for $p$-doping compressive strain has essentially no effect on $S$, whereas tensile strain enhances it considerably. For $n$-doping strain effects are always negligible for high carrier concentrations. For low doping, $\sigma / \tau$ varies with the strain inversely to $S$, whereas the strain dependence is completely lost above electron/hole concentrations of about $10^{19} \mathrm{~cm}^{-3}$. For $p$-doping, tensile strain affects the value of $S^{2} \sigma / \tau$ more than compressive strain, while for $n$-doping any strain effect is small. In general, for $n$-doping compressive strain is advantageous and for $p$-doping tensile strain, where stronger effects are observed in the latter case.

Fig. 3 shows $S, \sigma / \tau$, and $S^{2} \sigma / \tau$ at an elevated temperature of $500 \mathrm{~K}$ for both $n$ - and $p$-doping as a function of the carrier concentration. In each case, we find smaller values for $S$ and a shift of the maxima towards higher carrier concentrations as compared to the results at $300 \mathrm{~K}$ (as expected, since more carriers are available). Consequently, $S^{2} \sigma / \tau$ is enhanced at high doping. At a hole concentration of $7 \times 10^{20} \mathrm{~cm}^{-3}$, where the highest values of $S^{2} \sigma / \tau$ are obtained, $S$ and $\sigma / \tau$ are hardly modified by tensile strain. Hence, we can conclude that even a moderate tensile strain is sufficient to achieve a high $S^{2} \sigma / \tau$ value at $500 \mathrm{~K}$ for $p$-doping. For $n$-doping, on the other hand, compressive and tensile strain hardly enhance $S^{2} \sigma / \tau$. The temperature dependencies of $S$ and $S^{2} \sigma / \tau$ under strain at a fixed $p$-doping of $7 \times 10^{20} \mathrm{~cm}^{-3}$ are addressed in Fig. 4. We observe an almost linear increase with the temperature, except for the case of $2 \%$ tensile strain where we have a smaller band gap and reach the metallic regime above $550 \mathrm{~K}$.

To estimate the power factor and figure of merit, we obtain a relaxation time of $\tau=1.27 \times 10^{-14} \mathrm{~s}$ by comparing the calculated value of $\sigma / \tau=2 \times 10^{17}(\Omega \mathrm{ms})^{-1}$ without strain to the experimental $\sigma=2540(\Omega \mathrm{m})^{-1}$ (Ref. 15) and $\kappa=2.75 \mathrm{~W} / \mathrm{mK}$ of $\mathrm{Bi}_{1.98} \mathrm{Ca}_{0.02} \mathrm{Se}_{3}{ }^{7}$ at $300 \mathrm{~K}$. For $n$-doping, a maximal room temperature power factor of $12.5 \mu \mathrm{W} / \mathrm{cm} \mathrm{K}^{2}$ is predicted for unstrained $\mathrm{Bi}_{1.67} \mathrm{Tl}_{0.33} \mathrm{Se}_{3}$, which exceeds the experimental values of bulk $\mathrm{Bi}_{2} \mathrm{Se}_{3}\left(7.9 \mu \mathrm{W} / \mathrm{cm} \mathrm{K}^{2}\right)$ and $\mathrm{Bi}_{1.9} \mathrm{Tl}_{0.1} \mathrm{Se}_{3}\left(11 \mu \mathrm{W} / \mathrm{cm} \mathrm{K}{ }^{2}\right){ }^{15}$ We next estimate the power factor and figure of merit for temperatures of $300 \mathrm{~K}$ and $500 \mathrm{~K}$ under $2 \%$ tensile strain for the $p$-doping level at that $S^{2} \sigma / \tau$ is maximal. This results in a power factor of $31 \mu \mathrm{W} / \mathrm{cm} \mathrm{K}^{2}$ at $300 \mathrm{~K}$, which is slightly smaller than the value of bulk $\mathrm{Bi}_{2} \mathrm{Te}_{3}\left(40 \mu \mathrm{W} / \mathrm{cm} \mathrm{K}^{2}\right.$ (Ref. 29)) and grows to $48 \mu \mathrm{W} / \mathrm{cm} \mathrm{K}^{2}$ at $500 \mathrm{~K}$. Finally, a maximal figure of merit of 0.86 is obtained at $500 \mathrm{~K}$ for a hole concentration of $7 \times 10^{20} \mathrm{~cm}^{-3}$, which is about twice the value at $300 \mathrm{~K}$. We note that nanostructuring can be employed to further reduce the thermal conductivity, as reported for $\mathrm{Bi}_{2} \mathrm{Se}_{3} .{ }^{30}$

Tl-doping modifies the band gap without changing the effective masses at the conduction band minimum and valence band maximum (though a strong momentum-dependent spinorbit splitting is observed, since $\mathrm{Tl}$ is a heavy element). By analogous calculations, we find that both $\mathrm{Pb}$ - and $\mathrm{Cu}$-doping of $\mathrm{Bi}_{2} \mathrm{Se}_{3}$ result in a metallic nature. This can be explain by a
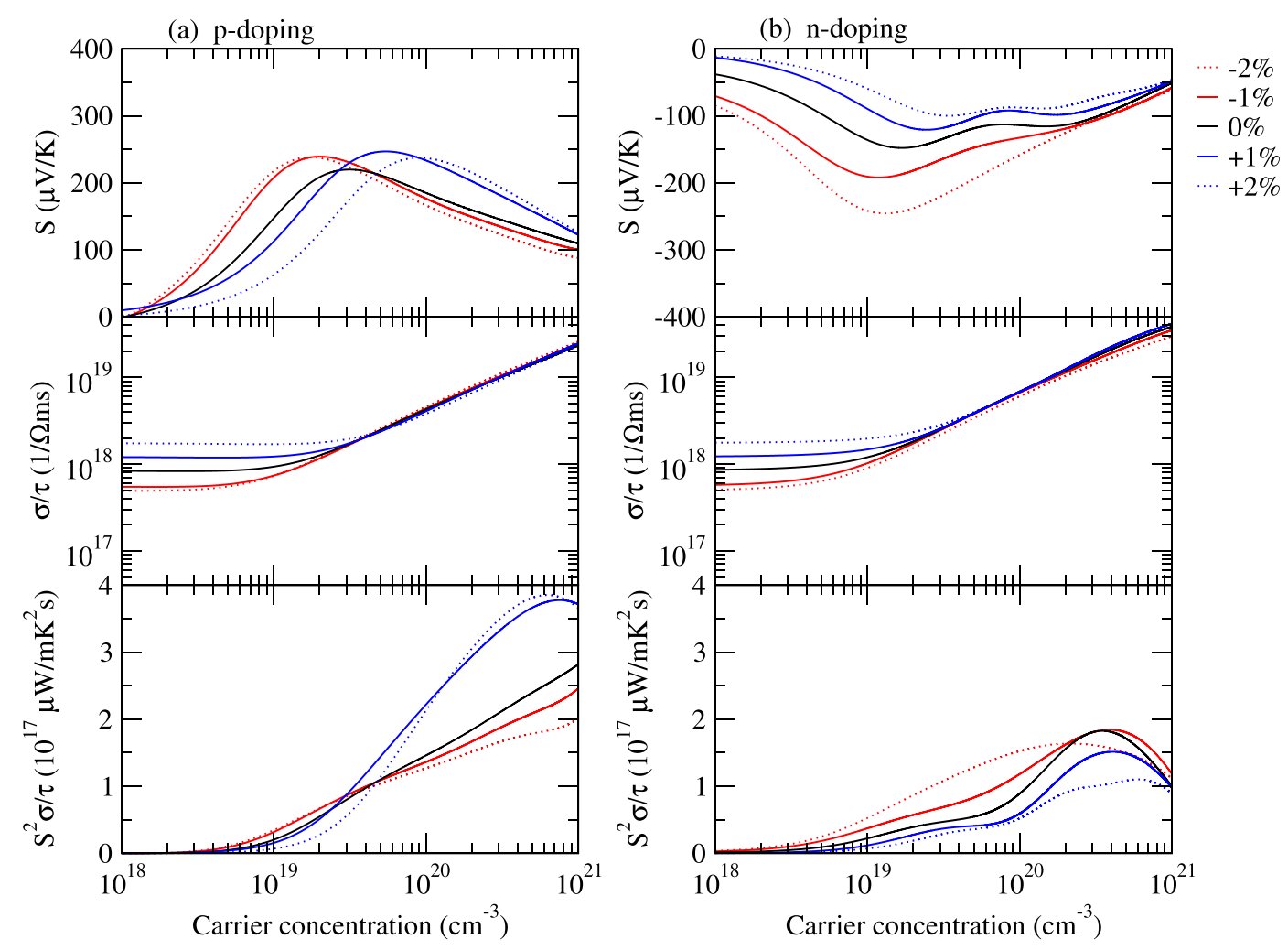

FIG. 3. $500 \mathrm{~K}: S, \sigma / \tau$, and $S^{2} \sigma / \tau$ under strain as a function of the carrier concentration. 


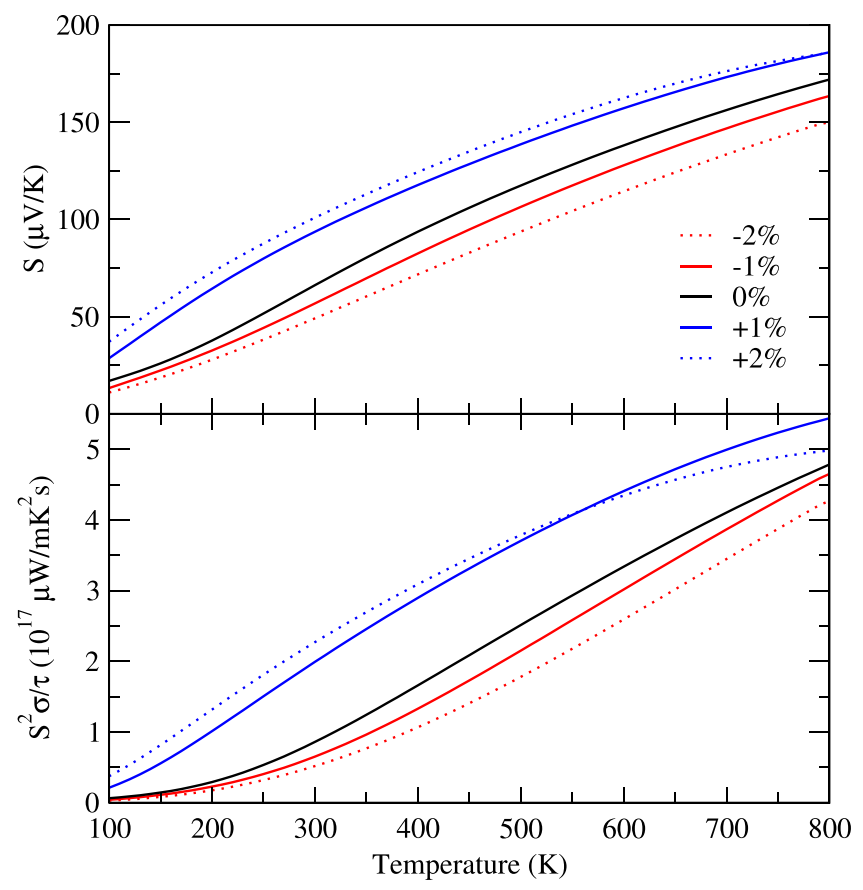

FIG. 4. $S$ and $S^{2} \sigma / \tau$ under strain as a function of the temperature for a fixed $p$-doping of $7 \times 10^{20} \mathrm{~cm}^{-3}$.

larger electronegativity difference between $\mathrm{Bi}(2.02)$ and $\mathrm{Tl}$ (1.62) as compared to that between $\mathrm{Bi}$ and $\mathrm{Pb}(2.33)$ or $\mathrm{Cu}$ (1.90). As a consequence, $\mathrm{Tl}$ donates more charge to $\mathrm{Bi}$ and $\mathrm{Se}$ as compared to $\mathrm{Pb}$ and $\mathrm{Cu}$ and therefore does not contribute to the band edges, in contrast to $\mathrm{Pb}$ and $\mathrm{Cu}$. Within the IIIA group of the periodic table, the band gap is found to increase from $\mathrm{Tl}$ to $\mathrm{In}, \mathrm{Ga}$, and Al-doping, whereas the dispersion does not change qualitatively, because the electronegativities are similar. However, due to the larger band gaps, strain in these cases is inefficient for tuning the thermoelectric properties, similar to pristine $\mathrm{Bi}_{2} \mathrm{Se}_{3}{ }^{13} \mathrm{Tl}$-doping therefore provides a unique combination of effects on the electronic structure that enables strain engineering.

In conclusion, the theoretical results obtained in this study from first principles can explain the experimental finding that the power factor is enhanced for increasing Tl-doping of $\mathrm{Bi}_{2} \mathrm{Se}_{3},{ }^{15}$ because the band gap is reduced while the effective mass of the charge carriers at the band edges is maintained. This effect of $\mathrm{Tl}$ is found to be exceptional under the possible dopants. Moderate strain cannot be used to enhance the power factor of pristine $\mathrm{Bi}_{2} \mathrm{Se}_{3}$, because the band gap is $300 \mathrm{meV}$ and therefore very large with respect to the thermal energy. After Tl-doping, on the contrary, strain turns out to be a very efficient tool for optimizing the power factor, because of the reduction of the band gap to $158 \mathrm{meV}$. We have demonstrated that in Tl-doped $\mathrm{Bi}_{2} \mathrm{Se}_{3}$ the band gap decreases monotonously from compressive to tensile strain. For high carrier concentrations tensile strain enhances $S$ more for $p$-doping than for $n$-doping due to the different dispersions of the valence and conduction bands. The maximal power factor $\left(48 \mu \mathrm{W} / \mathrm{cm} \mathrm{K}^{2}\right)$ and figure of the merit $(0.86)$ are obtained for a $p$-doping of $7 \times 10^{20} \mathrm{~cm}^{-3}$ under $2 \%$ tensile strain.

Research reported in this publication was supported by the King Abdullah University of Science and Technology (KAUST). We thank KAUST research computing for supplying computational resources. N.S. acknowledges SABIC for financial support.

${ }^{1}$ L. E. Bell, Science 321, 1457 (2008).

${ }^{2}$ R. Venkatasubramanian, E. Siivola, T. Colpitts, and B. O'Quinn, Nature 413, 597 (2001).

${ }^{3}$ T. C. Harman, P. J. Taylor, M. P. Walsh, and B. E. LaForge, Science 297, 2229 (2002).

${ }^{4}$ A. Soni, Y. Shen, M. Yin, Y. Zhao, L. Yu, X. Hu, Z. Dong, K. A. Khor, M. S. Dresselhaus, and Q. Xiong, Nano Lett. 12, 4305 (2012).

${ }^{5}$ A. H. Reshak, S. A. Khan, and S. Auluck, J. Mater. Chem. C 2, 2346 (2014).

${ }^{6}$ S. Nakajima, J. Phys. Chem. Solids. 24, 479 (1963).

${ }^{7}$ Y. S. Hor, A. Richardella, P. Roushan, Y. Xia, J. G. Checkelsky, A. Yazdani, M. Z. Hasan, N. P. Ong, and R. J. Cava, Phys. Rev. B 79, 195208 (2009).

${ }^{8}$ L. Xue, P. Zhou, C. X. Zhang, C. Y. He, G. L. Hao, L. Z. Sun, and J. X. Zhong, AIP Adv. 3, 052105 (2013).

${ }^{9}$ D. Kong, K. J. Koski, J. J. Cha, S. S. Hong, and Y. Cui, Nano Lett. 13, 632 (2013).

${ }^{10}$ C. Martin, V. Craciun, K. H. Miller, B. Uzakbaiuly, S. Buvaev, H. Berger, A. F. Hebard, and D. B. Tanner, Phys. Rev. B 87, 201201(R) (2013).

${ }^{11}$ J. Horák, P. Lošták, and A. Montaner, Phys. Status Solidi B 119, K17 (1983).

${ }^{12}$ P. Lošták, L. Beneš, S. Civiš, and H. Süssmann, J. Mater. Sci. 25, 277 (1990).

${ }^{13}$ X. Luo, M. B. Sullivan, and S. Y. Quek, Phys. Rev. B 86, 184111 (2012).

${ }^{14}$ Y. Saeed, N. Singh, and U. Schwingenschlögl, Appl. Phys. Lett. 104, 033105 (2014).

${ }^{15}$ P. Janíček, Č. Drašar, L. Beneš, and P. Lošták, Cryst. Res. Technol. 44, 505 (2009).

${ }^{16}$ H. Chi, W. Liu, K. Sun, X. Su, G. Wang, P. Lošták, V. Kucek, Č. Drašar, and C. Uher, Phys. Rev. B 88, 045202 (2013).

${ }^{17}$ S. Grimme, J. Comput. Chem. 27, 1787 (2006).

${ }^{18}$ P. Giannozzi, S. Baroni, N. Bonini, M. Calandra, R. Car, C. Cavazzoni, D. Ceresoli, G. L. Chiarotti, M. Cococcioni, I. Dabo, A. Dal Corso, S. de Gironcoli, S. Fabris, G. Fratesi, R. Gebauer, U. Gerstmann, C. Gougoussis, A. Kokalj, M. Lazzeri, L. Martin-Samos, N. Marzari, F. Mauri, R. Mazzarello, S. Paolini, A. Pasquarello, L. Paulatto, C. Sbraccia, S. Scandolo, G. Sclauzero, A. P. Seitsonen, A. Smogunov, P. Umari, and R. M. Wentzcovitch, J. Phys.: Condens. Matter 21, 395502 (2009).

${ }^{19}$ G. K. H. Madsen and D. J. Singh, Comput. Phys. Commun. 175, 67 (2006).

${ }^{20}$ G. K. H. Madsen, K. Schwarz, P. Blaha, and D. J. Singh, Phys. Rev. B 68, 125212 (2003).

${ }^{21}$ L. Zhang, M.-H. Du, and D. J. Singh, Phys. Rev. B 81, 075117 (2010).

${ }^{22}$ K. P. Ong, D. J. Singh, and P. Wu, Phys. Rev. B 83, 115110 (2011).

${ }^{23}$ T. J. Scheidemantel, C. Ambrosch-Draxl, T. Thonhauser, J. V. Badding, and J. O. Sofo, Phys. Rev. B 68, 125210 (2003).

${ }^{24}$ T. Thonhauser, T. J. Scheidemantel, J. O. Sofo, J. V. Badding, and G. D. Mahan, Phys. Rev. B 68, 085201 (2003).

${ }^{25}$ B. Y. Yavorsky, N. F. Hinsche, I. Mertig, and P. Zahn, Phys. Rev. B 84, 165208 (2011).

${ }^{26}$ J. Black, E. M. Conwell, L. Seigle, and C. W. Spencer, J. Phys. Chem. Solids 2, 240 (1957).

${ }^{27}$ J. C. Zheng, Front. Phys. China 3, 269 (2008).

${ }^{28}$ A. F. Ioffe, Semiconductor Thermoelements and Thermoelectric Cooling Information (London, UK, 1957).

${ }^{29}$ T. Caillat, M. Carle, P. Pierrat, H. Scherrer, and S. Scherrer, J. Phys. Chem. Solids 53, 1121 (1992).

${ }^{30}$ K. Kadel, L. Kumari, W.-Z. Li, J. Y. Huang, and P. P. Provencio, Nanoscale Res. Lett. 6, 57 (2011). 